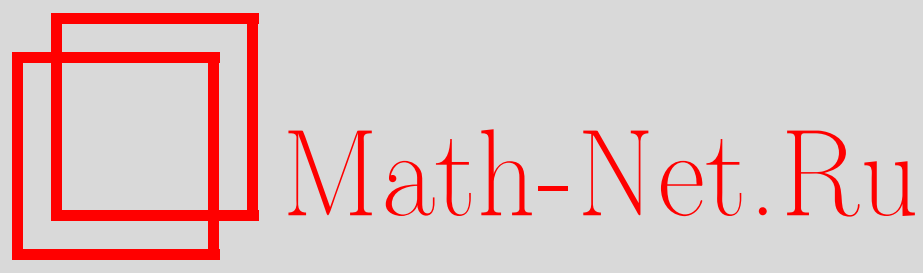

Р. Р. Мецаев, БРСТ-инвариантное эффективное действие источников, конформные поля и АдС/КТПсоответствие, ТМФ, 2014, том 181, номер 3, 495-514

DOI: https://doi.org/10.4213/tmf8759

Использование Общероссийского математического портала Math-Net.Ru подразумевает, что вы прочитали и согласны с пользовательским соглашением http: //www . mathnet.ru/rus/agreement

Параметры загрузки:

IP : 35.173 .219 .149

26 апреля 2023 г., 18:13:52

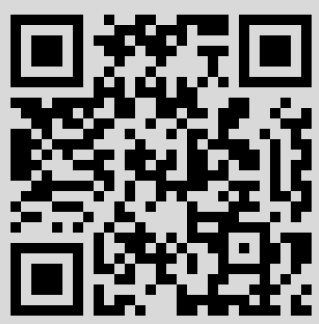




\title{
ФИЗИКА
}

Том 181, № 3

декабрь, 2014

\section{БРСТ-ИНВАРИАНТНОЕ ЭФФЕКТИВНОЕ ДЕЙСТВИЕ ИСТОЧНИКОВ, КОНФОРМНЫЕ ПОЛЯ И АДС/КТП-СООТВЕТСТВИЕ}

\begin{abstract}
Рассмотрены полностью симметричные безмассовые и массивные поля произвольного спина, распространяющиеся в пространстве анти-де Ситтера. Для таких полей с использованием калибровки типа де Дондера получены лагранжианы, инвариантные относительно глобальных БРСТ-преобразований. Эти лагранжианы используются для вычисления вакуумной статистической суммы и эффективного действия. Показано, что эффективное действие, вычисленное для ненормируемого решения уравнений движения поля с граничной задачей Дирихле, совпадает с БРСТ-инвариантным эффективным действием для источника. В случае безмассовых полей логарифмическая расходимость эффективного действия приводит к простому выражения для БРСТ-инвариантного лагранжиана конформного поля произвольного спина. Показано, что полям Наканиши-Лаутрупа, которые появляются в БРСТ-инвариантном действии конформных полей, можно дать геометрическую интерпретацию как граничных значений безмассовых полей в пространстве анти-де Ситтера.
\end{abstract}

Ключевые слова: БРСТ-симметрия, конформные поля, АдС/КТП-соответствие.

DOI: $10.4213 / \operatorname{tmf} 8759$

\section{1. ВВЕДЕНИЕ}

Тождества Славнова-Тейлора [1] и БРСТ-подход [2] играют важную роль в исследованиях проблемы перенормировок калибровочных теорий. Так как в квантовой теории поля уравнениям Гейзенберга трудно придать хорошо определенный смысл, основным объектом исследований в квантовой теории поля является $S$-матрица. Именно тождества Славнова-Тейлора и БРСТ-подход позволяют релятивистски-инвариантным образом исследовать процедуру устранения ультрафиолетовых расходимостей и построения перенормированной $S$-матрицы. При этом $S$-матрица получается не в результате априори заданной вычислительной схемы, а в результате реализации самосогласованной процедуры вычислений и предписаний, необходимых для получения унитарной $S$-матрицы. В определенном смысле можно сказать, что

*Физический институт им. П. Н. Лебедева РАН, Москва, Россия. E-mail: metsaev@lpi.ru 
процедура построения $S$-матрицы - это и есть способ определения и построения квантовой теории поля.

В настоящее время понятие $S$-матрицы определено для квантовой теории поля в плоском пространстве-времени. Обобщение понятия $S$-матрицы на квантовую теорию поля в пространстве анти-де Ситтера (АдС) оказывается проблематичным. Однако существует точка зрения, что роль $S$-матрицы в пространстве АдС могло бы играть так называемое эффективное действие источников. Такое действие определяется через континуальный интеграл для полей в пространстве АдС с заданными граничными значениями на границе пространства АдС. Граничные значения полей определяются как источники, и поэтому континуальный интеграл задает эффективное действие как функционал источников. Вариационные производные этого эффективного действия по источникам определяют корреляционные функции конформной теории поля, которая согласно гипотезе Малдасены дуальна теории поля в пространстве АдС. Таким образом, можно надеяться, что задачу построения полного квантового эффективного действия источников можно рассматривать как способ определения квантовой теории поля в пространстве АдС. По этой причине задача исследования эффективного действия источников представляется актуальной.

В квадратичном приближении по источникам древесное эффективное действие для источников спина $s=1$ и спина $s=2$ было изучено в работах [3]-[6], а для источника произвольного спина $s-$ в работах [7], [8]. Теоретико-групповые вопросы, связанные с проблемой вычисления эффективного действия, рассматривались в работах [9]-[12]. Эффективное действие для источника спина $s$, найденное в работах [7], [8], выражено в терминах полей, на которые наложены дифференциальные связи. В разделе 3 настоящей работы, введя источники для полей Фаддеева-Попова, мы находим БРСТ-инвариантное эффективное действие для источника спина $s$, которое выражено в терминах полей, свободных от дифференциальных связей.

Эффективное действие для источников, которые дуальны безмассовым полям в пространстве АдС, имеет логарифмическую расходимость. Как было показано в работе [4] для спина $s=2$ и в работе [7] для произвольного спина $s$, логарифмическая расходимость эффективного действия оказывается действием конформного поля спина $s=2$ и произвольного спина $s$ соответственно. В разделе 4 настоящей работы мы демонстрируем, что логарифмическая расходимость БРСТ-инвариантного эффективного действия источника, дуального безмассовому полю в пространстве АдС, оказывается БРСТ-инвариантным действием конформных полей. Это дает интересную возможность дать полям Наканиши-Лаутрупа, которые появляются в БРСТ-инвариантном действии конформных полей, геометрическую интерпретацию как граничных значений безмассовых полей в пространстве АдС.

В последнее время некоторое внимание привлекла задача вычисления однопетлевой вакуумной статистической суммы теории полей в пространстве АдС (см. [13], [14]). Так как БРСТ-инвариантный лагранжиан полей в пространстве АдС дает систематическую процедуру для вычисления вакуумной статистической суммы, мы начинаем наше обсуждение в разделе 2 с построения БРСТ-инвариантного лагранжиана полей в пространстве АдС и на основе этого вычисляем вакуумную статистическую сумму. 


\section{2. $S O(D, 1)$-КОВАРИАНТНЫЙ ПОДХОД И ВАКУУМНАЯ СТАТИСТИЧЕСКАЯ СУММА}

В этом разделе мы даем обзор $s o(d, 1)$-ковариантной лагранжевой формулировки динамики свободных полей в пространстве $(\mathrm{A}) \mathrm{C}_{d+1}$, развитой в работах [15], [16]. Эту формулировку мы будем использовать для построения БРСТ-инвариантного лагранжиана и вывода однопетлевой вакуумной статистической суммы.

В рамках метрического формализма для калибровочно-инвариантного описания безмассового поля спина $s$ в пространстве $(\mathrm{A}) \mathrm{C}_{d+1}$ используется дважды бесследовое тензорное поле алгебры $s o(d, 1)$ ранга $s$,

$$
\phi^{A_{1} \ldots A_{s}}, \quad \phi^{A A B B A_{5} \ldots A_{s}}=0, \quad s \geqslant 4 .
$$

Для калибровочно-инвариантного описания массивного поля спина $s$ в пространстве $\mathrm{AдC}_{d+1}$ используются следующие скалярное, векторное и дважды бесследовые тензорные поля алгебры $s o(d, 1)$ :

$$
\begin{array}{ll}
\phi^{A_{1} \ldots A_{s^{\prime}}}, & s^{\prime}=0,1, \ldots, s, \\
\phi^{A A B B A_{5} \ldots A_{s^{\prime}}}=0, & s^{\prime} \geqslant 4 .
\end{array}
$$

Все тензорные поля, приведенные в (2.1), (2.2), являются симметричными относительно перестановок своих тензорных индексов ${ }^{1)}$.

Вид калибровочно-инвариантного лагранжиана может быть упрощен, если использовать генерирующую форму калибровочных полей. Чтобы ввести генерирующую форму, введем осцилляторы $\alpha^{A}$, $\zeta$. С помощью таких осцилляторов поля, представленные в $(2.1),(2.2)$, могут быть упакованы в кет-векторы $|\phi\rangle$ следующим образом:

$$
\begin{aligned}
|\phi\rangle & =\frac{1}{s !} \alpha^{A_{1}} \ldots \alpha^{A_{s}} \phi^{A_{1} \ldots A_{s}}|0\rangle \\
|\phi\rangle & =\sum_{s^{\prime}=0}^{s} \frac{\zeta^{s-s^{\prime}}}{\sqrt{\left(s-s^{\prime}\right) !}}\left|\phi^{s^{\prime}}\right\rangle, \\
\left|\phi^{s^{\prime}}\right\rangle & =\frac{1}{s^{\prime} !} \alpha^{A_{1}} \ldots \alpha^{A_{s^{\prime}}} \phi^{A_{1} \ldots A_{s^{\prime}}}|0\rangle
\end{aligned}
$$

В терминах кет-векторов $|\phi\rangle$ калибровочно-инвариантный лагранжиан для безмассового и массивного полей может быть представлен единообразно:

$$
\begin{aligned}
\mathcal{L} & =\frac{1}{2} e\langle\phi|E| \phi\rangle, \\
E & =\boldsymbol{\mu}\left(\square_{(\mathrm{A}) \mathrm{dS}}+\mathbf{m}_{1}+\rho \boldsymbol{\alpha}^{2} \overline{\boldsymbol{\alpha}}^{2}\right)-\mathbf{L} \overline{\mathbf{L}}, \\
\overline{\mathbf{L}} & \equiv \overline{\boldsymbol{\alpha}} \mathbf{D}-\frac{1}{2} \boldsymbol{\alpha} \mathbf{D} \overline{\boldsymbol{\alpha}}^{2}-\overline{\mathbf{e}}_{1} \boldsymbol{\Pi}^{[1,2]}+\frac{1}{2} \mathbf{e}_{1} \overline{\boldsymbol{\alpha}}^{2}, \\
\mathbf{L} & \equiv \boldsymbol{\alpha} \mathbf{D}-\frac{1}{2} \boldsymbol{\alpha}^{2} \overline{\boldsymbol{\alpha}} \mathbf{D}-\mathbf{e}_{1} \boldsymbol{\Pi}^{[1,2]}+\frac{1}{2} \overline{\mathbf{e}}_{1} \boldsymbol{\alpha}^{2},
\end{aligned}
$$

\footnotetext{
1) В рамках метрического подхода безмассовые поля в $\mathrm{AдC}_{4}$ изучались в работе [17], а безмассовые поля в $\mathrm{AдC}_{d+1}, d \geqslant 3,-$ в работах [10], [18]. Состав полей в (2.2) для изучения массивных полей в $\mathrm{AдC}_{d+1}$ был предложен в работе [19]. В рамках реперного подхода поля в пространстве АдС были изучены в работах [20].
} 
где $\rho=\epsilon / R^{2}, \epsilon=1$ и $\epsilon=-1$ для пространств дС и АдС соответственно, а $R$ - радиус (А)дС, $e=\operatorname{det} e_{\underline{m}}^{A}, e_{\underline{m}}^{A}-$ базис реперов в пространстве (А)дС, $\square_{(\mathrm{A}) \mathrm{dS}}-$ оператор Даламбера в пространстве (А)дС, а $\mathbf{m}_{1}, \mathbf{e}_{1}, \overline{\mathbf{e}}_{1}$ даются выражениями

$$
\begin{aligned}
\mathbf{m}_{1}=\rho(s(s+d & -5)-2 d+4), \quad \mathbf{e}_{1}=0, \quad \overline{\mathbf{e}}_{1}=0 \quad \text { для безмассового поля; } \\
\mathbf{m}_{1} & =-m^{2}+\rho\left(s(s+d-5)-2 d+4+N_{\zeta}\left(2 s+d-1-N_{\zeta}\right)\right) \\
\mathbf{e}_{1} & =\zeta \mathbf{e}_{\zeta}, \quad \overline{\mathbf{e}}_{1}=-\mathbf{e}_{\zeta} \bar{\zeta} \quad \text { для массивного поля, } \\
\mathbf{e}_{\zeta} & \equiv\left(\frac{2 s+d-3-N_{\zeta}}{2 s+d-3-2 N_{\zeta}}\left(m^{2}-\rho N_{\zeta}\left(2 s+d-4-N_{\zeta}\right)\right)\right)^{1 / 2}
\end{aligned}
$$

Выражения для операторов $\boldsymbol{\alpha} \mathbf{D}, \boldsymbol{\alpha}^{2}, \boldsymbol{\mu}, \boldsymbol{\Pi}^{[1,2]}, N_{\zeta}$ приведены в приложении. Отметим, что в нашем подходе именно использование операторов $\mathbf{L}, \overline{\mathbf{L}}(2.7),(2.8)$ позволяет упростить структуру кинетического члена лагранжиана ${ }^{2)}$. Оператор $E(2.6)$ может быть альтернативно представлен следующим образом:

$$
E=\square(\mathrm{A}) \mathrm{dS}+M_{1}-\frac{1}{4} \boldsymbol{\alpha}^{2} \overline{\boldsymbol{\alpha}}^{2}\left(\square_{(\mathrm{A}) \mathrm{dS}}+M_{2}\right)-\mathbf{L} \overline{\mathbf{L}},
$$

где $M_{1}, M_{2}$ даются выражениями

$$
\begin{gathered}
M_{1}=\rho(s(s+d-5)-2 d+4), \quad \text { для безмассового поля; } \\
M_{2}=\rho(s(s+d-1)-6) \\
M_{1}=-m^{2}+\rho\left(s(s+d-5)-2 d+4+N_{\zeta}\left(2 s+d-1-N_{\zeta}\right)\right), \quad \text { для массивного поля. } \\
M_{2}=-m^{2}+\rho\left(s(s+d-1)-6+N_{\zeta}\left(2 s+d-5-N_{\zeta}\right)\right)
\end{gathered}
$$

Для описания калибровочных симметрий безмассового поля спина $s$ используется бесследовое тензорное поле алгебры $s o(d, 1)$ ранга $s-1$,

$$
\xi^{A_{1} \ldots A_{s-1}}, \quad \xi^{A A A_{3} \ldots A_{s-1}}=0, \quad s \geqslant 3,
$$

а для описания калибровочных симметрий массивного поля спина $s$ используются следующие скалярное, векторное и бесследовые тензорные поля алгебры $s o(d, 1)$ :

$$
\xi^{A_{1} \ldots A_{s^{\prime}}}, \quad s^{\prime}=0,1, \ldots, s-1, \quad \xi^{A A A_{3} \ldots A_{s^{\prime}}}=0, \quad s^{\prime} \geqslant 2 .
$$

Все тензорные поля, приведенные в (2.14), (2.15), являются симметричными относительно перестановок своих тензорных индексов.

Вид калибровочных преобразований может быть упрощен, если использовать генерирующую форму полей (2.14), (2.15):

$$
\begin{gathered}
|\xi\rangle=\frac{1}{(s-1) !} \alpha^{A_{1}} \ldots \alpha^{A_{s-1}} \xi^{A_{1} \ldots A_{s-1}}|0\rangle \quad \text { для безмассового поля; } \\
|\xi\rangle=\sum_{s^{\prime}=0}^{s-1} \frac{\zeta^{s-1-s^{\prime}}}{\sqrt{\left(s-1-s^{\prime}\right) !}}\left|\xi^{s^{\prime}}\right\rangle, \\
\left|\xi^{s^{\prime}}\right\rangle=\frac{1}{s^{\prime} !} \alpha^{A_{1}} \ldots \alpha^{A_{s^{\prime}}} \xi^{A_{1} \ldots A_{s^{\prime}}}|0\rangle
\end{gathered}
$$

${ }^{2)}$ Представление для лагранжиана в (2.5)-(2.8) было предложено в работах [15], [16]. Другие представления лагранжиана могут быть найдены в работах [18], [19]. 
В терминах кет-векторов $|\phi\rangle,|\xi\rangle$ калибровочные преобразования безмассового и массивного полей могут быть представлены единообразно:

$$
\delta|\phi\rangle=\mathbf{G}|\xi\rangle, \quad \mathbf{G} \equiv \boldsymbol{\alpha} \mathbf{D}-\mathbf{e}_{1}-\boldsymbol{\alpha}^{2} \frac{1}{2 N_{\boldsymbol{\alpha}}+d-1} \overline{\mathbf{e}}_{1},
$$

где операторы $\mathbf{e}_{1}, \overline{\mathbf{e}}_{1}$ приведены в $(2.9),(2.10)$, а $N_{\boldsymbol{\alpha}}$ определен в приложении.

\section{1. БРСТ-инвариантный лагранжиан и вакуумная статистическая} сумма. Для построения БРСТ-инвариантного лагранжиана следует ввести поля Фаддеева-Попова и Наканиши-Лаутрупа ${ }^{3)}$. Генерирующая форма полей ФаддееваПопова описывается кет-векторами $|c\rangle,|\bar{c}\rangle$, а генерирующая форма полей Наканиши-Лаутрупа описывается кет-вектором $|b\rangle$. Разложение этих кет-векторов в скалярные, векторные и тензорные поля алгебры $s o(d, 1)$ имеет вид

$$
\begin{gathered}
|c\rangle=\frac{1}{(s-1) !} \alpha^{A_{1}} \ldots \alpha^{A_{s-1}} c^{A_{1} \ldots A_{s-1}}|0\rangle, \quad|\bar{c}\rangle=\frac{1}{(s-1) !} \alpha^{A_{1}} \ldots \alpha^{A_{s-1}} \bar{c}^{A_{1} \ldots A_{s-1}}|0\rangle \\
|b\rangle=\frac{1}{(s-1) !} \alpha^{A_{1}} \ldots \alpha^{A_{s-1}} b^{A_{1} \ldots A_{s-1}}|0\rangle \quad \text { для безмассового поля; } \\
|c\rangle=\sum_{s^{\prime}=0}^{s-1} \frac{\zeta^{s-1-s^{\prime}}}{\sqrt{\left(s-1-s^{\prime}\right) !}}\left|c^{s^{\prime}}\right\rangle, \quad\left|c^{s^{\prime}}\right\rangle=\frac{1}{s^{\prime} !} \alpha^{A_{1}} \ldots \alpha^{A_{s^{\prime}}} c^{A_{1} \ldots A_{s^{\prime}}}|0\rangle \\
|\bar{c}\rangle=\sum_{s^{\prime}=0}^{s-1} \frac{\zeta^{s-1-s^{\prime}}}{\sqrt{\left(s-1-s^{\prime}\right) !}}\left|\bar{c}^{s^{\prime}}\right\rangle, \quad\left|\bar{c}^{s^{\prime}}\right\rangle=\frac{1}{s^{\prime} !} \alpha^{A_{1}} \ldots \alpha^{A_{s^{\prime}}} \bar{c}^{A_{1} \ldots A_{s^{\prime}}}|0\rangle \\
|b\rangle=\sum_{s^{\prime}=0}^{s-1} \frac{\zeta^{s-1-s^{\prime}}}{\sqrt{\left(s-1-s^{\prime}\right) !}}\left|b^{s^{\prime}}\right\rangle, \quad\left|b^{s^{\prime}}\right\rangle=\frac{1}{s^{\prime} !} \alpha^{A_{1}} \ldots \alpha^{A_{s^{\prime}}} b^{A_{1} \ldots A_{s^{\prime}}}|0\rangle
\end{gathered}
$$

для массивного поля.

Тензорные поля в (2.19), (2.20) являются бесследовыми симметричными тензорными полями алгебры $s o(d, 1)$. Используя язык кет-векторов, БРСТ-инвариантный лагранжиан $\mathcal{L}_{\text {tot }}$ в произвольной $\alpha$-калибровке можно записать следующим образом:

$$
\begin{aligned}
\mathcal{L}_{\mathrm{tot}} & =\mathcal{L}+\mathcal{L}_{\mathrm{qu}}, \\
\frac{1}{e} \mathcal{L}_{\mathrm{qu}} & =-\langle b|\overline{\mathbf{L}}| \phi\rangle+\left\langle\bar{c}\left|\left(\square_{(\mathrm{A}) \mathrm{dS}}+M_{\mathrm{FP}}\right)\right| c\right\rangle+\frac{1}{2} \alpha\langle b|| b\rangle, \\
M_{\mathrm{FP}} & \equiv-m^{2}+\rho\left((s-1)(s+d-2)+N_{\zeta}\left(2 s+d-3-N_{\zeta}\right)\right),
\end{aligned}
$$

где оператор $\overline{\mathbf{L}}$ определен в (2.7). Лагранжиан (2.21) инвариантен относительно следующих БРСТ- и анти-БРСТ-преобразований (инвариантность лагранжиана понимается по модулю полных производных):

$$
\begin{aligned}
& \mathbf{s}|\phi\rangle=\mathbf{G}|c\rangle, \quad \mathbf{s}|c\rangle=0, \quad \mathbf{s}|\bar{c}\rangle=|b\rangle, \quad \mathbf{s}|b\rangle=0, \\
& \overline{\mathbf{s}}|\phi\rangle=\mathbf{G}|\bar{c}\rangle, \quad \overline{\mathbf{s}}|c\rangle=-|b\rangle, \quad \overline{\mathbf{s}}|\bar{c}\rangle=0, \quad \overline{\mathbf{s}}|b\rangle=0,
\end{aligned}
$$

3) В настоящей работе мы исследуем глобальные БРСТ-симметрии. Изучение безмассовых и массивных полей с локальными БРСТ-симметриями можно найти в работах [21]-[30]. 
где оператор $\mathbf{G}$ определен в соотношении (2.18). БРСТ- и анти-БРСТ-преобразования (2.24), (2.25) нильпотентны вне массовой поверхности: $\mathrm{s}^{2}=0, \overline{\mathrm{s}}^{2}=0$, $\mathrm{s} \overline{\mathrm{s}}+\overline{\mathrm{s}} \mathrm{s}=0$.

Для вычисления вакуумной статистической суммы выбираем калибровку $\alpha=$ 1 и интегрируем по полю Наканиши-Лаутрупа. В результате лагранжиан (2.21) принимает вид

$$
\frac{1}{e} \mathcal{L}_{\text {tot }}=\frac{1}{2}\left\langle\phi\left|\boldsymbol{\mu}\left(\square_{(\mathrm{A}) \mathrm{dS}}+\mathbf{m}_{1}+\rho \boldsymbol{\alpha}^{2} \overline{\boldsymbol{\alpha}}^{2}\right)\right| \phi\right\rangle+\left\langle\bar{c}\left|\left(\square_{(\mathrm{A}) \mathrm{dS}}+M_{\mathrm{FP}}\right)\right| c\right\rangle .
$$

Напомним, что вакуумная статистическая сумма не зависит от выбора калибровки. Однако отметим что именно представление для лагранжиана в (2.5)-(2.8) и выбор калибровки $\alpha=1$ существенно упрощают структуру $\mathcal{L}_{\text {tot }}(2.26)$ и последующее вычисление вакуумной статистической суммы ${ }^{4)}$. Альтернативно лагранжиан (2.26) может быть переписан как

$$
\frac{1}{e} \mathcal{L}_{\text {tot }}=\frac{1}{2}\left\langle\phi\left|\left(\square_{(\mathrm{A}) \mathrm{dS}}+M_{1}-\frac{1}{4} \boldsymbol{\alpha}^{2} \overline{\boldsymbol{\alpha}}^{2}\left(\square_{(\mathrm{A}) \mathrm{dS}}+M_{2}\right)\right)\right| \phi\right\rangle+\left\langle\bar{c}\left|\left(\square+M_{\mathrm{FP}}\right)\right| c\right\rangle .
$$

Удобно также разложить дважды бесследовый кет-вектор $|\phi\rangle$ в два бесследовых кет-вектора $\left|\phi_{\mathrm{I}}\right\rangle,\left|\phi_{\mathrm{II}}\right\rangle$ следующим образом:

$$
\begin{array}{rlrl}
|\phi\rangle & =\left|\phi_{\mathrm{I}}\right\rangle+\boldsymbol{\alpha}^{2} \mathcal{N}\left|\phi_{\mathrm{II}}\right\rangle, \quad \overline{\boldsymbol{\alpha}}^{2}\left|\phi_{\mathrm{I}}\right\rangle=0, \quad \overline{\boldsymbol{\alpha}}^{2}\left|\phi_{\mathrm{II}}\right\rangle=0, & \\
\mathcal{N} & \equiv((2 s+d-3)(2 s+d-5))^{-1 / 2} & & \text { для безмассового поля; } \\
\mathcal{N} & \equiv\left(\left(2 s+d-3-2 N_{\zeta}\right)\left(2 s+d-5-2 N_{\zeta}\right)\right)^{-1 / 2} & & \text { для массивного поля. }
\end{array}
$$

Подставляя (2.28) в (2.27), получаем

$$
\begin{aligned}
\frac{1}{e} \mathcal{L}_{\text {tot }}= & \frac{1}{2}\left\langle\phi_{\mathrm{I}}\left|\left(\square_{(\mathrm{A}) \mathrm{dS}}+M_{1}\right)\right| \phi_{\mathrm{I}}\right\rangle-\frac{1}{2}\left\langle\phi_{\mathrm{II}}\left|\left(\square_{(\mathrm{A}) \mathrm{dS}}+M_{2}+4 \rho\right)\right| \phi_{\mathrm{II}}\right\rangle+ \\
& +\left\langle\bar{c}\left|\left(\square_{(\mathrm{A}) \mathrm{dS}}+M_{\mathrm{FP}}\right)\right| c\right\rangle .
\end{aligned}
$$

Разложение кет-векторов $\left|\phi_{\mathrm{I}}\right\rangle,\left|\phi_{\mathrm{II}}\right\rangle$ в тензорные поля имеет вид

$$
\begin{aligned}
\left|\phi_{\mathrm{I}}\right\rangle & =\frac{1}{s !} \alpha^{A_{1}} \ldots \alpha^{A_{s}} \phi_{\mathrm{I}}^{A_{1} \ldots A_{s}}|0\rangle, \\
\left|\phi_{\mathrm{II}}\right\rangle & =\frac{1}{(s-2) !} \alpha^{A_{1}} \ldots \alpha^{A_{s-2}} \phi_{\mathrm{II}}^{A_{1} \ldots A_{s-2}}|0\rangle \\
\left|\phi_{\mathrm{I}}\right\rangle & =\sum_{s^{\prime}=0}^{s} \frac{\zeta^{s-s^{\prime}}}{\sqrt{\left(s-s^{\prime}\right) !}}\left|\phi_{\mathrm{I}}^{s^{\prime}}\right\rangle, \quad\left|\phi_{\mathrm{II}}\right\rangle=\sum_{s^{\prime}=0}^{s-2} \frac{\zeta^{s-2-s^{\prime}}}{\sqrt{\left(s-2-s^{\prime}\right) !}}\left|\phi_{\mathrm{II}}^{s^{\prime}}\right\rangle, \\
\left|\phi_{\mathrm{I}, \mathrm{II}}^{s^{\prime}}\right\rangle & =\frac{1}{s^{\prime} !} \alpha^{A_{1}} \ldots \alpha^{A_{s^{\prime}}} \phi_{\mathrm{I}, \mathrm{II}}^{A_{1} \ldots A_{s^{\prime}}}|0\rangle \quad \text { для безмассового поля; }
\end{aligned}
$$

В терминах тензорных полей лагранжиан (2.31) может быть представлен следующим образом:

$$
\begin{array}{ll}
\mathcal{L}_{\text {tot }}=\mathcal{L}_{\mathrm{I}}^{s}-\mathcal{L}_{\mathrm{II}}^{s-2}+\mathcal{L}_{\mathrm{FP}}^{s-1} & \text { для безмассового поля, } \\
\mathcal{L}_{\text {tot }}=\sum_{s^{\prime}=0}^{s} \mathcal{L}_{\mathrm{I}}^{s^{\prime}}-\sum_{s^{\prime}=0}^{s-2} \mathcal{L}_{\mathrm{II}}^{s^{\prime}}+\sum_{s^{\prime}=0}^{s-1} \mathcal{L}_{\mathrm{FP}}^{s^{\prime}} & \text { для массивного поля, }
\end{array}
$$

4) Недавние исследования в работах [31] открывают новые интересные возможности доказательства независимости вакуумной статистической суммы от выбора калибровки. 


$$
\begin{aligned}
& \mathcal{L}_{\mathrm{I}, \mathrm{II}}^{s^{\prime}}=\frac{e}{2 s^{\prime} !} \phi_{\mathrm{I}, \mathrm{II}}^{A_{1} \ldots A_{s^{\prime}}}\left(\mathcal{D}^{2}+M_{1}^{s^{\prime}}\right) \phi_{\mathrm{I}, \mathrm{II}}^{A_{1} \ldots A_{s^{\prime}}}, \\
& \mathcal{L}_{\mathrm{FP}}^{s^{\prime}}=\frac{e}{s^{\prime} !} \bar{c}^{A_{1} \ldots A_{s^{\prime}}}\left(\mathcal{D}^{2}+M_{1}^{s^{\prime}}\right) c^{A_{1} \ldots A_{s^{\prime}}}, \\
& M_{1}^{s} \equiv \rho((s-2)(s+d-2)-s), \\
& M_{1}^{s-2} \equiv \rho(s(s+d-1)-2) \\
& M_{1}^{s^{\prime}} \equiv-m^{2}+\rho(2(s-1)(s+d-2)- \\
& \left.-s^{\prime}\left(s^{\prime}+d-1\right)\right) \\
& M_{1}^{s-1} \equiv \rho(s-1)(s+d-2), \\
& \text { для безмассового поля, } \\
& \text { для массивного поля. }
\end{aligned}
$$

Из выражений (2.34)-(2.38) видно, что вакуумная статистическая сумма дается выражениями

$$
\begin{aligned}
Z & =\frac{D^{s-1} D^{s-1}}{D^{s} D^{s-2}} \quad \text { для безмассового поля; } \\
Z & =\prod_{s^{\prime}=0}^{s-1} D^{s^{\prime}} D^{s^{\prime}}\left(\prod_{s^{\prime}=0}^{s} D^{s^{\prime}} \prod_{s^{\prime}=0}^{s-2} D^{s^{\prime}}\right)^{-1}=\frac{D^{s-1}}{D^{s}} \quad \text { для массивного поля, } \\
D^{s^{\prime}} & \equiv\left(\operatorname{det}\left(-\mathcal{D}^{2}-M_{1}^{s^{\prime}}\right)\right)^{1 / 2},
\end{aligned}
$$

где детерминант оператора Даламбера вычисляется на пространстве бесследового тензорного поля ранга $s^{\prime}$. Для конкретных вычислений удобно использовать следующее представление для $D^{s^{\prime}}(2.41)$ :

$$
D^{s^{\prime}}=D^{s^{\prime} \perp} D^{s^{\prime}-1}
$$

где $D^{s^{\prime} \perp}$ имеет тот же вид, что и в $(2.41)$, однако теперь детерминант оператора Даламбера вычисляется на пространстве бесследового и бездивергентного тензорного поля ранга $s^{\prime}$. Используя (2.42) в (2.39) и (2.40), находим

$$
\begin{aligned}
& Z=\frac{D^{s-1 \perp}}{D^{s \perp}} \quad \text { для безмассового поля; } \\
& Z=\frac{1}{D^{s \perp}} \quad \text { для массивного поля. }
\end{aligned}
$$

Статсуммы (2.43), (2.44) были получены ранее другими методами в работах [13], [14].

\section{3. $S O(D-1,1)$-КОВАРИАНТНЫЙ ПОДХОД И ЭФФЕКТИВНОЕ ДЕЙСТВИЕ}

В этом разделе мы даем обзор so $(d-1,1)$-ковариантной лагранжевой формулировки динамики свободных полей в пространстве $\mathrm{AдC}_{d+1}$, развитой в работах [15], [16]. Эту формулировку мы будем использовать для построения БРСТ-инвариантного лагранжиана и вычисления БРСТ-инвариантного древесного эффективного действия.

$s o(d-1,1)$-Ковариантная формулировка калибровочных полей в $\mathrm{AдC}_{d+1}$ основывается на использовании пуанкаре-параметризации пространства $\mathrm{AдC}_{d+1}$,

$$
d s^{2}=\frac{1}{z^{2}}\left(d x^{a} d x^{a}+d z d z\right) .
$$


Из (3.1) видно, что метрический тензор имеет явные симметрии $s o(d-1,1)$.

В рамках метрического формализма для калибровочно-инвариантного описания безмассового поля спина $s$ в пространстве $\mathrm{AдC}_{d+1}$ используются следующие скалярное, векторное и дважды бесследовые тензорные поля алгебры $s o(d-1,1)$ :

$$
\phi^{a_{1} \ldots a_{s^{\prime}}}, \quad s^{\prime}=0,1, \ldots, s, \quad \phi^{a a b b a_{5} \ldots a_{s^{\prime}}}=0, \quad s^{\prime} \geqslant 4
$$

Для калибровочно-инвариантного описания массивного поля спина $s$ в пространстве $\mathrm{AдC}_{d+1}$ используются следующие скалярные, векторные и дважды бесследовые тензорные поля алгебры $s o(d-1,1)$ :

$$
\phi_{\lambda}^{a_{1} \ldots a_{s^{\prime}}}, \quad s^{\prime}=0,1, \ldots, s, \quad \phi_{\lambda}^{a a b b a_{5} \ldots a_{s^{\prime}}}=0, \quad s^{\prime} \geqslant 4, \quad \lambda \in\left[s-s^{\prime}\right]_{2} .
$$

Здесь и далее соотношение $\lambda \in[n]_{2}$ означает, что $\lambda$ принимает значения $\lambda=-n$, $-n+2,-n+4, \ldots, n-4, n-2, n$. Тензорные поля, приведенные в (3.2), (3.3), являются симметричными относительно перестановок своих тензорных индексов.

Для упрощения вида калибровочно-инвариантного лагранжиана используем генерирующую форму калибровочных полей. Введя осцилляторы $\alpha^{a}, \alpha^{z}, \zeta$, замечаем, что поля (3.2), (3.3) могут быть упакованы в кет-векторы $|\phi\rangle$ следующим образом:

$$
\begin{gathered}
|\phi\rangle \equiv \sum_{s^{\prime}=0}^{s} \frac{\alpha_{z}^{s-s^{\prime}}}{\sqrt{\left(s-s^{\prime}\right) !}}\left|\phi^{s^{\prime}}\right\rangle, \\
\left|\phi^{s^{\prime}}\right\rangle \equiv \frac{1}{s^{\prime} !} \alpha^{a_{1}} \ldots \alpha^{a_{s^{\prime}}} \phi^{a_{1} \ldots a_{s^{\prime}}}|0\rangle \\
|\phi\rangle=\sum_{s^{\prime}=0}^{s}\left|\phi^{s^{\prime}}\right\rangle, \\
\left|\phi^{s^{\prime}}\right\rangle=\sum_{\lambda \in\left[s-s^{\prime}\right]_{2}} \frac{\zeta^{\left(s-s^{\prime}+\lambda\right) / 2} \alpha_{z}^{\left(s-s^{\prime}-\lambda\right) / 2} \alpha^{a_{1}} \ldots \alpha^{a_{s^{\prime}}}}{s^{\prime} ! \sqrt{\left(\left(s-s^{\prime}+\lambda\right) / 2\right) !\left(\left(s-s^{\prime}-\lambda\right) / 2\right) !}} \phi_{\lambda}^{a_{1} \ldots a_{s^{\prime}}}|0\rangle
\end{gathered}
$$

для массивного поля.

В терминах кет-векторов $|\phi\rangle(3.4),(3.5)$ калибровочно-инвариантное действие для безмассового и массивного полей может быть представлено единообразно:

$$
\begin{aligned}
S & =\int d^{d} x d z \mathcal{L}, \\
\mathcal{L} & =\frac{1}{2}\left\langle\phi\left|\mu\left(\square-\mathcal{M}^{2}\right)\right| \phi\right\rangle+\frac{1}{2}\langle\bar{L} \phi|| \bar{L} \phi\rangle, \\
\bar{L} & \equiv \bar{\alpha} \partial-\frac{1}{2} \alpha \partial \bar{\alpha}^{2}-\bar{e}_{1} \Pi^{[1,2]}+\frac{1}{2} e_{1} \bar{\alpha}^{2},
\end{aligned}
$$

$\square \equiv \partial^{a} \partial^{a}, \partial^{a}=\eta^{a b} \partial / \partial x^{b},|\bar{L} \phi\rangle \equiv \bar{L}|\phi\rangle$. Выражения для скалярных произведений $\alpha \partial$, $\alpha^{2}, \mu, \Pi^{[1,2]}$ приведены в приложении. Бра-векторы $\langle\phi|,\langle\bar{L} \phi|$ определяются в соответствии с правилом $\langle\phi| \equiv(|\phi\rangle)^{\dagger},\langle\bar{L} \phi| \equiv(|\bar{L} \phi\rangle)^{\dagger}$. Выражения для операторов $\mathcal{M}^{2}$, $e_{1}, \bar{e}_{1}$ приведены в табл. 1. Из (3.7) видно, что кинетические члены безмассового и массивного полей имеют одну и ту же зависимость от векторных осцилляторов $\alpha^{a}$ и от производных $\partial^{a}$. Из табл. 1 видно, что вся зависимость кинетических членов 
безмассового и массивного полей от скалярных осцилляторов $\zeta, \alpha^{z}$, от радиальной координаты $z$ и от производной $\partial_{z}=\partial / \partial z$ задается операторами $\mathcal{M}^{2}, e_{1}, \bar{e}_{1}$.

Для описания калибровочных симметрий следует ввести параметры калибровочных преобразований. Генерирующая форма параметров калибровочных преобразований описывается кет-векторами $|\xi\rangle$. Разложение этих кет-векторов в скалярные, векторные и симметричные тензорные поля алгебры $s o(d-1,1)$ имеет вид

$$
\begin{gathered}
|\xi\rangle \equiv \sum_{s^{\prime}=0}^{s-1} \frac{\alpha_{z}^{s-1-s^{\prime}}}{\sqrt{\left(s-1-s^{\prime}\right) !}}\left|\xi^{s^{\prime}}\right\rangle, \quad \text { для безмассового поля; } \\
\left|\xi^{s^{\prime}}\right\rangle \equiv \frac{1}{s^{\prime} !} \alpha^{a_{1}} \ldots \alpha^{a_{s^{\prime}} \xi^{a_{1} \ldots a_{s^{\prime}}}|0\rangle} \\
|\xi\rangle=\sum_{s^{\prime}=0}^{s-1}\left|\xi^{s^{\prime}}\right\rangle, \\
\left|\xi^{s^{\prime}}\right\rangle=\sum_{\lambda \in\left[s-1-s^{\prime}\right]_{2}} \frac{\zeta^{\left(s-1-s^{\prime}+\lambda\right) / 2} \alpha_{z}^{\left(s-1-s^{\prime}-\lambda\right) / 2} \alpha^{a_{1}} \ldots \alpha^{a_{s^{\prime}}}}{s^{\prime} ! \sqrt{\left(\left(s-1-s^{\prime}+\lambda\right) / 2 r\right) !\left(\left(s-1-s^{\prime}-\lambda\right) / 2\right) !}} \xi_{\lambda}^{a_{1} \ldots a_{s^{\prime}}}|0\rangle
\end{gathered}
$$

для массивного поля.

Тензорные поля в (3.9), (3.10) являются бесследовыми тензорами алгебры $s o(d-1,1)$.

В терминах кет-векторов $|\phi\rangle,|\xi\rangle$ калибровочные преобразования безмассового и массивного полей могут быть представлены единообразно:

$$
\delta|\phi\rangle=G|\xi\rangle, \quad G \equiv \alpha \partial-e_{1}-\alpha^{2} \frac{1}{2 N_{\alpha}+d-2} \bar{e}_{1},
$$

где операторы $e_{1}, \bar{e}_{1}$ приведены в табл. 1 , а определение $N_{\alpha}$ дано в приложении.

\section{1. БРСТ-инвариантный лагранжиан и эффективное действие источ-} ников. Для построения БРСТ-инвариантного лагранжиана следует ввести поля Фаддеева-Попова и Наканиши-Лаутрупа. Генерирующая форма полей ФаддееваПопова описывается кет-векторами $|c\rangle,|\bar{c}\rangle$, а генерирующая форма полей НаканишиЛаутрупа описывается кет-вектором $|b\rangle$. Разложение этих кет-векторов в соответствующие скалярные, векторные и тензорные поля $\bar{c}^{a_{1} \ldots a_{s^{\prime}}}, c^{a_{1} \ldots a_{s^{\prime}}}, b^{a_{1} \ldots a_{s^{\prime}}}$ имеет тот же вид (3.9), (3.10) и получается простой заменой в (3.9), (3.10)

$$
\xi \rightarrow c, \quad \xi \rightarrow \bar{c}, \quad \xi \rightarrow b .
$$

Тензорные поля, возникающие в разложении кет-векторов $|c\rangle,|\bar{c}\rangle,|b\rangle$, являются бесследовыми симметричными тензорными полями алгебры $s o(d-1,1)$. С использованием языка кет-векторов БРСТ-инвариантный лагранжиан $\mathcal{L}_{\text {tot }}$ в произвольной $\alpha$-калибровке может быть записан следующим образом:

$$
\mathcal{L}_{\text {tot }}=\mathcal{L}+\mathcal{L}_{\text {qu }}, \quad \mathcal{L}_{\text {qu }}=-\langle b|\bar{L}| \phi\rangle+\left\langle\bar{c}\left|\left(\square-\mathcal{M}^{2}\right)\right| c\right\rangle+\frac{1}{2} \alpha\langle b|| b\rangle,
$$

где оператор $\bar{L}$ определен в (3.8), а оператор $\mathcal{M}^{2}$ приведен в табл. 1. Лагранжиан (3.13) инвариантен относительно следующих БРСТ- и анти-БРСТ-преобразований:

$$
\mathrm{s}|\phi\rangle=G|c\rangle, \quad \mathrm{s}|c\rangle=0, \quad \mathrm{~s}|\bar{c}\rangle=|b\rangle, \quad \mathrm{s}|b\rangle=0,
$$


ТАБлицА 1. Операторы $\mathcal{M}^{2}, e_{1}, \bar{e}_{1}$, задающие лагранжиан, калибровочные преобразования и БРСТ-преобразования полей в пространстве АдС в рамках $s o(d-1,1)$-ковариантной формулировки $(m$ - массовый параметр массивного поля). Также приведены операторы $e_{1}, \bar{e}_{1}$, задающие БРСТ-преобразования канонических и аномальных источников.

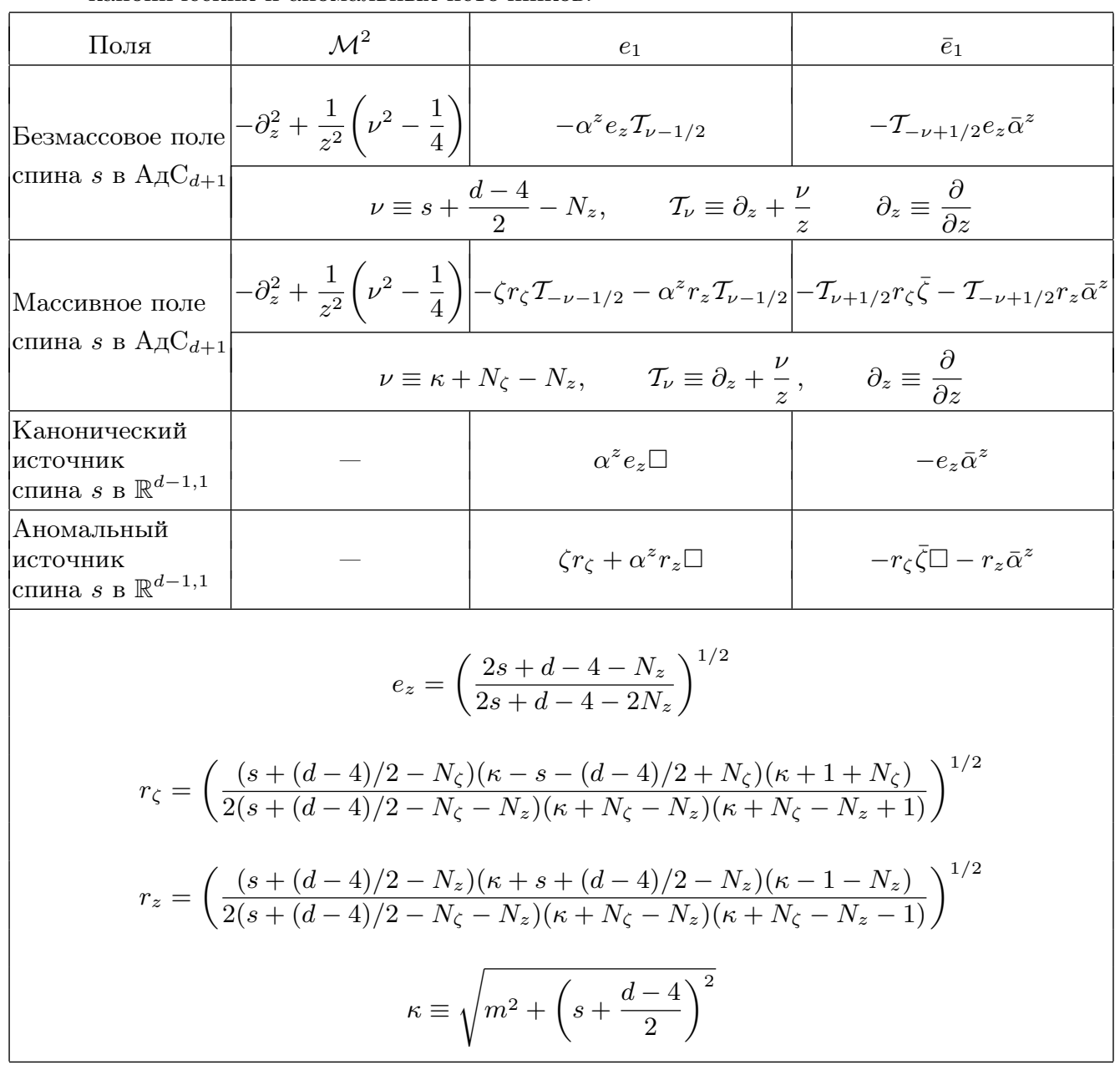

$$
\overline{\mathbf{s}}|\phi\rangle=G|\bar{c}\rangle, \quad \overline{\mathbf{s}}|c\rangle=-|b\rangle, \quad \overline{\mathbf{s}}|\bar{c}\rangle=0, \quad \overline{\mathbf{s}}|b\rangle=0,
$$

где оператор $G$ приведен в (3.11). БРСТ- и анти-БРСТ-преобразования (3.14), (3.15) нильпотентны вне массовой поверхности: $\mathrm{s}^{2}=0, \overline{\mathrm{s}}^{2}=0, \mathrm{~s} \overline{\mathrm{s}}+\overline{\mathrm{s}} \mathrm{s}=0$.

В дальнейшем для вычисления эффективного действия источников удобно выбрать калибровку $\alpha=1$ и проинтегрировать по полям Наканиши-Лаутрупа. В результате БРСТ-инвариантный лагранжиан (3.13) принимает вид

$$
\mathcal{L}_{\text {tot }}=\frac{1}{2}\left\langle\phi\left|\mu\left(\square-\mathcal{M}^{2}\right)\right| \phi\right\rangle+\left\langle\bar{c}\left|\left(\square-\mathcal{M}^{2}\right)\right| c\right\rangle
$$


БРСТ- и анти-БРСТ-симметрии лагранжиана (3.16) реализуются следующими преобразованиями:

$$
\begin{aligned}
& \mathrm{s}|\phi\rangle=G|c\rangle, \quad \mathrm{s}|c\rangle=0, \quad \mathrm{~s}|\bar{c}\rangle=\bar{L}|\phi\rangle, \\
& \overline{\mathrm{s}}|\phi\rangle=G|\bar{c}\rangle, \quad \overline{\mathrm{s}}|c\rangle=-\bar{L}|\phi\rangle, \quad \overline{\mathrm{s}}|\bar{c}\rangle=0,
\end{aligned}
$$

где операторы $\bar{L}$ и $G$ приведены в (3.8) и (3.11) соответственно. Преобразования (3.17), (3.18) нильпотентны только на массовой поверхности полей Фаддеева-Попова.

Для реализации АдС/КТП-соответствия следует решить две задачи. Во-первых, следует найти решения уравнений движения полей с определенными граничными условиями для полей, т. е. решить задачу Дирихле. Граничные условия выбираются из требования, чтобы граничные значения полей отвечали некоторым определенным представлениям конформной алгебры, которые называются источниками. Отметим, что граничным значениям безмассовых полей отвечают представления конформной алгебры, которые называются каноническими источниками, а граничным значениям массивных полей отвечают представления конформной алгебры, которые называются аномальными источниками. Во-вторых, следует подставить решение задачи Дирихле в действие. Действие, вычисленное при решении задачи Дирихле, называется эффективным действием. В случае безмассовых полей эффективное действие является функционалом канонических источников, а в случае массивных полей эффективное действие является функционалом аномальных источников. Вторая вариационная производная эффективного действия представляет собой корреляционную функцию операторов конформной теории поля. Займемся вычислением эффективного действия.

Уравнения движения для калибровочных полей и полей Фаддеева-Попова, которые следуют из лагранжиана (3.16), имеют следующий вид:

$$
\square_{\nu}|\phi\rangle=0, \quad \square_{\nu}|\bar{c}\rangle=0, \quad \square_{\nu}|c\rangle=0, \quad \square_{\nu} \equiv \square+\partial_{z}^{2}-\frac{1}{z^{2}}\left(\nu^{2}-\frac{1}{4}\right) .
$$

Решение задачи Дирихле для уравнений движений (3.19) с граничными значениями, отвечающими источникам калибровочных полей и полей Фаддеева-Попова, может быть представлено в виде

$$
\begin{aligned}
|\phi(x, z)\rangle & =\sigma_{\nu} \int d^{d} y G_{\nu}(x-y, z)\left|\phi_{\mathrm{sh}}(y)\right\rangle, \\
|c(x, z)\rangle & =\sigma_{\nu} \int d^{d} y G_{\nu}(x-y, z)\left|c_{\mathrm{sh}}(y)\right\rangle, \\
|\bar{c}(x, z)\rangle & =\sigma_{\nu} \int d^{d} y G_{\nu}(x-y, z)\left|\bar{c}_{\mathrm{sh}}(y)\right\rangle, \\
\sigma_{\nu} & \equiv \frac{2^{\nu} \Gamma(\nu)}{2^{\bar{\kappa}} \Gamma(\bar{\kappa})}(-1)^{N_{z}},
\end{aligned}
$$

где функция Грина дается выражением

$$
G_{\nu}(x, z)=\frac{c_{\nu} z^{\nu+1 / 2}}{\left(z^{2}+|x|^{2}\right)^{\nu+d / 2}}, \quad c_{\nu} \equiv \frac{\Gamma(\nu+d / 2)}{\pi^{d / 2} \Gamma(\nu)}
$$


а кет-векторы $\left|\phi_{\mathrm{sh}}\right\rangle,\left|c_{\mathrm{sh}}\right\rangle,\left|\bar{c}_{\mathrm{sh}}\right\rangle$ в правых частях обозначают кет-векторы источников калибровочных полей и полей Фаддеева-Попова. Из выражений, приведенных в (3.20), а также из асимптотического поведения функции Грина

$$
G_{\nu}(x, z) \stackrel{z \rightarrow 0}{\longrightarrow} z^{-\nu+1 / 2} \delta^{d}(x)
$$

получаем асимптотическое поведение решения задачи Дирихле для калибровочных полей

$$
|\phi(x, z)\rangle \stackrel{z \rightarrow 0}{\longrightarrow} z^{-\nu+1 / 2} \sigma_{\nu}\left|\phi_{\mathrm{sh}}(x)\right\rangle
$$

и аналогичное поведение для полей Фаддеева-Попова.

Следующим шагом в реализации АдС/КТП-соответствия является подстановка решения (3.20) в действие (3.6) с лагранжианом (3.16). Строго говоря, при этом к действию следует добавить граничный член, который, очевидно, не влияет на уравнения движения, но, как правило, влияет на значение эффективного действия для источников. Метод нахождения граничного члена был предложен в работе [32]. Следуя этому методу, можно убедиться, что выражение для лагранжиана, учитывающее вклад граничного члена, имеет следующий вид:

$$
\mathcal{L}_{\text {tot }}=\frac{1}{2}\left\langle\partial^{a} \phi|\mu| \partial^{a} \phi\right\rangle+\frac{1}{2}\left\langle\mathcal{T}_{\nu-1 / 2} \phi|\mu| \mathcal{T}_{\nu-1 / 2} \phi\right\rangle+\left\langle\partial^{a} \bar{c}|| \partial^{a} c\right\rangle+\left\langle\mathcal{T}_{\nu-1 / 2} \bar{c}|| \mathcal{T}_{\nu-1 / 2} c\right\rangle,
$$

где $\mathcal{T}_{\nu}$ приведено в табл. 1. При переходе от (3.16) к (3.24) мы также перешли к евклидовой сигнатуре, что означает замену $\mathcal{L} \rightarrow-\mathcal{L}$. Нетрудно убедиться, что на решениях уравнений движения действие (3.6) с лагранжианом (3.24) может быть представлено следующим образом ${ }^{5)}$ :

$$
-S_{\text {tot }}^{\mathrm{eff}}=\left.\int d^{d} x \mathcal{L}_{\text {tot }}^{\mathrm{eff}}\right|_{z \rightarrow 0}, \quad \mathcal{L}_{\text {tot }}^{\mathrm{eff}}=\frac{1}{2}\left\langle\phi\left|\mu \mathcal{T}_{\nu-1 / 2}\right| \phi\right\rangle+\left\langle\bar{c}\left|\mathcal{T}_{\nu-1 / 2}\right| c\right\rangle .
$$

Подставляя (3.20) в (3.25), получаем следующее эффективное действие:

$$
-S_{\text {tot }}^{\mathrm{eff}}=2 \bar{\kappa} c_{\bar{\kappa}} \Gamma_{\text {tot }},
$$

где функционал $\Gamma_{\text {tot }}$ имеет вид

$$
\begin{aligned}
\Gamma_{\text {tot }} & \equiv \int d^{d} x_{1} d^{d} x_{2} \mathcal{L}_{\text {tot }}^{12}, \\
\mathcal{L}_{\text {tot }}^{12} & \equiv \frac{1}{2}\left\langle\phi_{\mathrm{sh}}\left(x_{1}\right)\left|\frac{\mu f_{\nu}}{\left|x_{12}\right|^{2 \nu+d}}\right| \phi_{\mathrm{sh}}\left(x_{2}\right)\right\rangle+\left\langle\bar{c}_{\mathrm{sh}}\left(x_{1}\right)\left|\frac{f_{\nu}}{\left|x_{12}\right|^{2 \nu+d}}\right| c_{\mathrm{sh}}\left(x_{2}\right)\right\rangle, \\
f_{\nu} & \equiv \frac{\Gamma(\nu+d / 2) \Gamma(\nu+1)}{4^{\bar{\kappa}-\nu} \Gamma(\bar{\kappa}+d / 2) \Gamma(\bar{\kappa}+1)}, \quad\left|x_{12}\right|^{2} \equiv x_{12}^{a} x_{12}^{a}, \quad x_{12}^{a}=x_{1}^{a}-x_{2}^{a}, \\
\nu & \equiv s+\frac{d-4}{2}-N_{z}, \quad \bar{\kappa} \equiv s+\frac{d-4}{2} \quad \text { для канонических источников, } \\
\nu & \equiv \kappa+N_{\zeta}-N_{z}, \quad \bar{\kappa} \equiv \kappa \quad \text { для аномальных источников, }
\end{aligned}
$$

5) Мы используем лагранжев подход для изучения АдС/КТП-соответствия. Исследование АдС/КТП-соответствия на основе симметрий теории высших спинов можно найти в работах [33]. 
а операторы $N_{\zeta}, N_{z}, \mu$ определены в приложении. Напомним также, что $c_{\kappa}$ определено в (3.21), а параметр $\kappa$ приведен в табл. 1.

Эффективное действие (3.26) инвариантно относительно следующих БРСТ- и анти-БРСТ-преобразований:

$$
\begin{gathered}
\mathrm{s}\left|\phi_{\mathrm{sh}}\right\rangle=G\left|c_{\mathrm{sh}}\right\rangle, \quad \mathrm{s}\left|c_{\mathrm{sh}}\right\rangle=0, \quad \mathrm{~s}\left|\bar{c}_{\mathrm{sh}}\right\rangle=\bar{L}\left|\phi_{\mathrm{sh}}\right\rangle, \\
\overline{\mathrm{s}}\left|\phi_{\mathrm{sh}}\right\rangle=G\left|\bar{c}_{\mathrm{sh}}\right\rangle, \quad \overline{\mathrm{s}}\left|c_{\mathrm{sh}}\right\rangle=-\bar{L}\left|\phi_{\mathrm{sh}}\right\rangle, \quad \overline{\mathrm{s}}\left|\bar{c}_{\mathrm{sh}}\right\rangle=0, \\
G \equiv \alpha \partial-e_{1}-\alpha^{2} \frac{1}{2 N_{\alpha}+d-2} \bar{e}_{1}, \quad \bar{L} \equiv \bar{\alpha} \partial-\frac{1}{2} \alpha \partial \bar{\alpha}^{2}-\bar{e}_{1} \Pi^{[1,2]}+\frac{1}{2} e_{1} \bar{\alpha}^{2},
\end{gathered}
$$

где операторы $e_{1}, \bar{e}_{1}$, отвечающие каноническим и аномальным источникам, приведены в табл. 1. БРСТ- и анти-БРСТ-преобразования, приведенные в (3.32), (3.33), нильпотентны.

\section{4. КОНФОРМНЫЕ ПОЛЯ}

Для канонических источников ядро эффективного действия (3.27) не определено при четных значениях $d$ (см. [34]). Этому ядру можно придать смысл, используя размерную регуляризацию. При этом оказывается, что при снятии регуляризации ядро имеет логарифмическую расходимость. Ниже мы демонстрируем, что эта логарифмическая расходимость оказывается БРСТ-инвариантным действием конформных полей.

Введем параметр регуляризации $\varepsilon$ :

$$
d-[d]=-2 \varepsilon, \quad[d] \text { четное, }
$$

где $[d]$ - целая часть $d$. Используя (4.1) и выражение для $\nu$, приведенное в (3.30), отмечаем, что при малых $\varepsilon$ ядро имеет следующее асимптотическое поведение:

$$
\frac{1}{|x|^{2 \nu+d}} \stackrel{\varepsilon \sim 0}{\sim} \frac{1}{\varepsilon} \varrho_{\nu} \square^{\nu} \delta(x), \quad \varrho_{\nu} \equiv \frac{\pi^{d / 2}}{4^{\nu} \Gamma(\nu+1) \Gamma(\nu+d / 2)} .
$$

Подставляя (4.2) в выражение для $\Gamma_{\text {tot }}(3.27)$, получаем

$$
\begin{gathered}
\Gamma_{\text {tot }} \stackrel{\varepsilon \sim 0}{\sim} \frac{1}{\varepsilon} \varrho_{\nu_{s}} \int d^{d} x \mathcal{L}_{\text {tot }} \quad \text { для канонических источников, } \\
\mathcal{L}_{\text {tot }}=\frac{1}{2}\left\langle\phi\left|\mu \square^{\nu}\right| \phi\right\rangle+\left\langle\bar{c}\left|\square^{\nu}\right| c\right\rangle, \quad \nu \equiv s+\frac{d-4}{2}-N_{z} .
\end{gathered}
$$

При переходе от (3.28) к (4.3) использовались упрощающие обозначения идентификации кет-векторов: $|\phi\rangle \equiv\left|\phi_{\mathrm{sh}}\right\rangle,|\bar{c}\rangle \equiv\left|\bar{c}_{\mathrm{sh}}\right\rangle,|c\rangle \equiv\left|c_{\mathrm{sh}}\right\rangle$. Лагранжиан (4.3) является БРСТ-инвариантным лагранжианом конформного поля спина $s^{6)}$. Для демонстрационных целей рассмотрим лагранжиан для полей спина $s=1,2$ и произвольного $s$ по отдельности.

\footnotetext{
6) Мы интересуемся глобальными БРСТ-симметриями свободных конформных полей. Изучение свободных конформных полей с локальными БРСТ-симметриями можно найти в работе [35]. Обсуждение общей структуры теории взаимодействующих конформных полей в трехмерном пространстве можно найти в работе [36].
} 
4.1. Конформное поле спина $s=1$. Для конформного поля спина $s=1$ лагранжиан (4.3) принимает вид

$$
\mathcal{L}_{\text {tot }}=\frac{1}{2} \phi^{a} \square^{k+1} \phi^{a}+\frac{1}{2} \phi \square^{k} \phi+\bar{c} \square^{k+1} c, \quad k \equiv \frac{d-4}{2} .
$$

Лагранжиан (4.4) инвариантен относительно БРСТ- и анти-БРСТ-преобразований

$$
\begin{aligned}
& \mathbf{s} \phi^{a}=\partial^{a} c, \quad \mathrm{~s} \phi=-\square c, \quad \mathrm{~s} c=0, \quad \mathrm{~s} \bar{c}=\partial^{a} \phi^{a}+\phi, \\
& \overline{\mathbf{s}} \phi^{a}=\partial^{a} \bar{c}, \quad \overline{\mathbf{s}} \phi=-\square \bar{c}, \quad \overline{\mathbf{s}} c=-\partial^{a} \phi^{a}-\phi, \quad \overline{\mathrm{s}} \bar{c}=0 .
\end{aligned}
$$

Нетрудно проверить, что БРСТ-преобразования (4.5) нильпотентны вне массовой поверхности. Это связано с тем, что скалярное поле $\phi$ играет роль поля Наканиши-Лаутрупа. Действительно, вводя вместо поля $\phi$ новое поле $b$ с помощью замены

$$
b=\phi+\partial^{a} \phi^{a}
$$

получаем следующее выражение для лагранжиана (4.4):

$$
\begin{aligned}
\mathcal{L}_{\text {tot }} & =\mathcal{L}+\mathcal{L}_{\mathrm{qu}}, \\
\mathcal{L} & =-\frac{1}{4} F^{a b} \square^{k} F^{a b}, \quad F^{a b} \equiv \partial^{a} \phi^{b}-\partial^{b} \phi^{a}, \\
\mathcal{L}_{\mathrm{qu}} & =-b \square^{k} \partial^{a} \phi^{a}+\frac{1}{2} b \square^{k} b+\bar{c} \square^{k+1} c,
\end{aligned}
$$

в то время как БРСТ- и анти-БРСТ-преобразования (4.5), (4.6) принимают вид

$$
\begin{array}{llll}
\mathrm{s} \phi^{a}=\partial^{a} c, & \mathrm{~s} b=0, & \mathrm{~s} c=0, & \mathrm{~s} \bar{c}=b, \\
\overline{\mathrm{s}} \phi^{a}=\partial^{a} \bar{c}, & \overline{\mathrm{s}} b=0, & \overline{\mathrm{s}} c=-b, & \overline{\mathrm{s}} \bar{c}=0 .
\end{array}
$$

Из (4.10), (4.11), (4.12) видно, что поле $b$ действительно является полем Наканиши-Лаутрупа. Отметим также, что только для $d=4$ поле $b$ может быть исключено из лагранжиана, если использовать уравнения движения для этого поля.

В рамках АдС/КТП-соответствия поля $\phi^{a}$, $\phi$ появляются как граничные значения ненормируемого решения уравнений движений безмассовых полей в пространстве АдС. По этой причине соотношение (4.7) можно рассматривать как геометрическую интерпретацию поля Наканиши-Лаутрупа.

4.2. Конформное поле спина $s=2$. Для конформного поля спина $s=2$ лагранжиан (4.3) принимает вид

$$
\begin{gathered}
\mathcal{L}_{\text {tot }}=\frac{1}{4} \phi^{a b} \square^{k+1} \phi^{a b}-\frac{1}{8} \phi^{a a} \square^{k+1} \phi^{b b}+\frac{1}{2} \phi^{a} \square^{k} \phi^{a}+\frac{1}{2} \phi \square^{k-1} \phi+ \\
+\bar{c}^{a} \square^{k+1} c^{a}+\bar{c} \square^{k} c, \quad k \equiv \frac{d-2}{2} .
\end{gathered}
$$


Лагранжиан (4.13) инвариантен относительно БРСТ-преобразований

$$
\begin{aligned}
\mathrm{s} \phi^{a b} & =\partial^{a} c^{b}+\partial^{b} c^{a}+\frac{2}{d-2} \eta^{a b} c, \\
\mathrm{~s} \phi^{a} & =\partial^{a} c-\square c^{a}, \\
\mathrm{~s} \phi & =-u \square c, \\
\mathrm{~s} c^{a} & =0, \quad \mathrm{~s} c=0, \\
\mathrm{~s} \bar{c}^{a} & =\partial^{b} \phi^{a b}-\frac{1}{2} \partial^{a} \phi^{b b}+\phi^{a}, \\
\mathrm{~s} \bar{c} & =\partial^{a} \phi^{a}+\frac{1}{2} \square \phi^{a a}+u \phi, \quad u \equiv\left(2 \frac{d-1}{d-2}\right)^{1 / 2} .
\end{aligned}
$$

Анти-БРСТ-преобразования получаются из (4.14) простыми подстановками $\mathrm{s} \rightarrow \overline{\mathrm{s}}$, $c \rightarrow \bar{c}, \bar{c} \rightarrow-c$. БРСТ-преобразования (4.14) нильпотентны вне массовой поверхности. Это связано с тем, что векторное поле $\phi^{a}$ и скалярное поле $\phi$ играют роль полей Наканиши-Лаутрупа. Чтобы продемонстрировать это, явно введем вместо $\phi^{a}, \phi$ новые поля $b^{a}, b$ следующим образом:

$$
b^{a}=\partial^{b} \phi^{a b}-\frac{1}{2} \partial^{a} \phi^{b b}+\phi^{a}, \quad b=\partial^{a} \phi^{a}+\frac{1}{2} \square \phi^{a a}+u \phi .
$$

Обращая соотношения (4.15):

$$
\phi^{a}=b^{a}-\partial^{b} \phi^{a b}+\frac{1}{2} \partial^{a} \phi^{b b}, \quad u \phi=b-\partial^{a} b^{a}+\partial^{a} \partial^{b} \phi^{a b}-\square \phi^{a a},
$$

и подставляя $\phi^{a}, \phi(4.16)$ в (4.13), получаем следующее выражение для лагранжиана (4.13):

$$
\begin{aligned}
\mathcal{L}_{\text {tot }}= & \mathcal{L}+\mathcal{L}_{\mathrm{qu}}, \\
\mathcal{L}= & R_{\text {lin }}^{a b} \square^{k-1} R_{\text {lin }}^{a b}-\frac{d}{4(d-1)} R_{\text {lin }} \square^{k-1} R_{\text {lin }}, \\
\mathcal{L}_{\mathrm{qu}}=- & b^{a} \square^{k}\left(\partial^{b} \phi^{a b}-\frac{1}{2} \partial^{a} \phi^{b b}\right)+\frac{1}{u^{2}}\left(b-\partial^{a} b^{a}\right) \square^{k-1}\left(\partial^{c} \partial^{e} \phi^{c e}-\square \phi^{c c}\right)+ \\
& \quad+\frac{1}{2} b^{a} \square^{k} b^{a}+\frac{1}{2 u^{2}}\left(b-\partial^{a} b^{a}\right) \square^{k-1}\left(b-\partial^{c} b^{c}\right)+\bar{c}^{a} \square^{k+1} c^{a}+\bar{c} \square^{k} c,
\end{aligned}
$$

где $R_{\text {lin }}^{a b}, R_{\text {lin }}$ означают линеаризованные тензор Риччи и скаляр Риччи соответственно,

$$
\begin{aligned}
R_{\operatorname{lin}}^{a b} & =\frac{1}{2}\left(-\square \phi^{a b}+\partial^{a} \partial^{c} \phi^{c b}+\partial^{b} \partial^{c} \phi^{c a}-\partial^{a} \partial^{b} \phi^{c c}\right), \\
R_{\operatorname{lin}} & =\partial^{a} \partial^{b} \phi^{a b}-\square \phi^{a a} .
\end{aligned}
$$

Используя БРСТ-преобразования (4.14) и соотношения (4.16), легко получаем

$$
\mathrm{s} \bar{c}^{a}=b^{a}, \quad \mathrm{~s} \bar{c}=b, \quad \mathrm{~s} b^{a}=0, \quad \mathrm{~s} b=0 .
$$

Из (4.17), (4.21) видно, что поля $b^{a}, b$ действительно являются полями Наканиши-Лаутрупа. 
4.3. Конформное поле спина $s$. Для случая конформного поля спина $s$ генерирующая форма БРСТ-инвариантного лагранжиана приведена в (4.3). БРСТ- и анти-БРСТ-преобразования, относительно которых лагранжиан (4.3) инвариантен, имеют вид

$$
\begin{array}{lll}
\mathrm{s}|\phi\rangle=G|c\rangle, & \mathrm{s}|c\rangle=0, & \mathrm{~s}|\bar{c}\rangle=\bar{L}|\phi\rangle, \\
\overline{\mathrm{s}}|\phi\rangle=G|\bar{c}\rangle, & \overline{\mathrm{s}}|c\rangle=-\bar{L}|\phi\rangle, & \overline{\mathrm{s}}|\bar{c}\rangle=0,
\end{array}
$$

где операторы $G, \bar{L}$ задаются соотношениями

$$
\begin{aligned}
G & \equiv \alpha \partial-e_{1}-\alpha^{2} \frac{1}{2 N_{\alpha}+d-2} \bar{e}_{1}, \\
\bar{L} & \equiv \bar{\alpha} \partial-\frac{1}{2} \alpha \partial \bar{\alpha}^{2}-\bar{e}_{1} \Pi^{[1,2]}+\frac{1}{2} e_{1} \bar{\alpha}^{2}, \\
e_{1} & =\alpha^{z} e_{z} \square, \quad \bar{e}_{1}=-e_{z} \bar{\alpha}^{z}, \quad e_{z}=\left(\frac{2 s+d-4-N_{z}}{2 s+d-4-2 N_{z}}\right)^{1 / 2},
\end{aligned}
$$

а оператор $\Pi^{[1,2]}$ определен в приложении. Нетрудно проверить, что БРСТ- и анти-БРСТ-преобразования (4.22), (4.23) нильпотентны вне массовой поверхности. В данном случае роль поля Наканиши-Лаутрупа играет кет-вектор $|b\rangle$, определяемый соотношением $|b\rangle \equiv \bar{L}|\phi\rangle$. Это нетрудно проверить, используя преобразования (4.22), (4.23), которые приводят к желаемым соотношениям $\mathrm{s} \bar{c}=|b\rangle, \mathrm{s}|b\rangle=0$, $\overline{\mathbf{s}}|c\rangle=-|b\rangle, \overline{\mathbf{s}}|b\rangle=0$.

Чтобы проиллюстрировать структуру лагранжиана (4.3), используем разложение кет-векторов в скалярные, векторные и тензорные поля алгебры $s o(d-1,1)$,

$$
\begin{aligned}
|\phi\rangle & =\sum_{s^{\prime}=0}^{s} \frac{\alpha_{z}^{s-s^{\prime}}}{\sqrt{\left(s-s^{\prime}\right) !}}\left|\phi^{s^{\prime}}\right\rangle, & \left|\phi^{s^{\prime}}\right\rangle & \equiv \frac{1}{s^{\prime} !} \alpha^{a_{1}} \ldots \alpha^{a_{s^{\prime}}} \phi^{a_{1} \ldots a_{s^{\prime}}}|0\rangle, \\
|c\rangle & =\sum_{s^{\prime}=0}^{s-1} \frac{\alpha_{z}^{s-1-s^{\prime}}}{\sqrt{\left(s-1-s^{\prime}\right) !}}\left|c^{s^{\prime}}\right\rangle, & \left|c^{s^{\prime}}\right\rangle & \equiv \frac{1}{s^{\prime} !} \alpha^{a_{1}} \ldots \alpha^{a_{s^{\prime}}} c^{a_{1} \ldots a_{s^{\prime}}}|0\rangle, \\
|\bar{c}\rangle & =\sum_{s^{\prime}=0}^{s-1} \frac{\alpha_{z}^{s-1-s^{\prime}}}{\sqrt{\left(s-1-s^{\prime}\right) !}}\left|\bar{c} s^{s^{\prime}}\right\rangle, & \left|\bar{c}^{s^{\prime}}\right\rangle & \equiv \frac{1}{s^{\prime} !} \alpha^{a_{1}} \ldots \alpha^{a_{s^{\prime}}} \bar{c}^{a_{1} \ldots a_{s^{\prime}}}|0\rangle .
\end{aligned}
$$

Заметим, что калибровочные тензорные поля в (4.25) являются дважды бесследовыми тензорами алгебры $s o(d-1,1)$, а тензорные поля Фаддеева-Попова в $(4.26),(4.27)$ являются бесследовыми тензорами алгебры $s o(d-1,1)$. Подстановка кет-векторов (4.25)-(4.27) в (4.3) приводит к следующему представлению для лагранжиана (4.3):

$$
\begin{aligned}
\mathcal{L}_{\mathrm{tot}} & =\sum_{s^{\prime}=0}^{s} \mathcal{L}^{s^{\prime}}+\sum_{s^{\prime}=0}^{s-1} \mathcal{L}_{\mathrm{FP}}^{s^{\prime}}, \\
\mathcal{L}^{s^{\prime}} & =\frac{1}{2 s^{\prime} !}\left(\phi^{a_{1} \ldots a_{s^{\prime}}} \square^{\nu_{s^{\prime}}} \phi^{a_{1} \ldots a_{s^{\prime}}}-\frac{s^{\prime}\left(s^{\prime}-1\right)}{4} \phi^{a a a_{3} \ldots a_{s^{\prime}}} \square^{\nu_{s^{\prime}}} \phi^{b b a_{3} \ldots a_{s^{\prime}}}\right), \\
\mathcal{L}_{\mathrm{FP}}^{s^{\prime}} & =\frac{1}{s^{\prime} !} \bar{c}^{a_{1} \ldots a_{s^{\prime}}} \square^{\nu_{s^{\prime}}+1} c^{a_{1} \ldots a_{s^{\prime}}}, \quad \nu_{s^{\prime}}=s^{\prime}+\frac{d-4}{2} .
\end{aligned}
$$


Для вычисления вакуумной статистической суммы удобно разложить каждый дважды бесследовый тензор $\phi^{a_{1} \ldots a_{s^{\prime}}}$ на два бесследовых тензора. С этой целью разложим дважды бесследовый кет-вектор $|\phi\rangle$ в два бесследовых кет-вектора $\left|\phi_{\mathrm{I}}\right\rangle,\left|\phi_{\mathrm{II}}\right\rangle$ :

$$
\begin{gathered}
|\phi\rangle=\left|\phi_{\mathrm{I}}\right\rangle+\alpha^{2} \mathcal{N}\left|\phi_{\mathrm{II}}\right\rangle, \quad \bar{\alpha}^{2}\left|\phi_{\mathrm{I}}\right\rangle=0, \quad \bar{\alpha}^{2}\left|\phi_{\mathrm{II}}\right\rangle=0 \\
\mathcal{N} \equiv\left(\left(2 s+d-4-2 N_{z}\right)\left(2 s+d-6-2 N_{z}\right)\right)^{-1 / 2}
\end{gathered}
$$

Подставляя (4.29) в (4.3), получаем

$$
\mathcal{L}_{\text {tot }}=\frac{1}{2}\left\langle\phi_{\mathrm{I}}\left|\square^{\nu}\right| \phi_{\mathrm{I}}\right\rangle-\frac{1}{2}\left\langle\phi_{\mathrm{II}}\left|\square^{\nu}\right| \phi_{\mathrm{II}}\right\rangle+\left\langle\bar{c}\left|\square^{\nu}\right| c\right\rangle
$$

Разложение кет-векторов $\left|\phi_{\mathrm{I}, \mathrm{II}}\right\rangle$ в бесследовые тензорные поля $\phi_{\mathrm{I}, \mathrm{II}}^{a_{1} \ldots a_{s^{\prime}}}$ имеет вид

$$
\begin{gathered}
\left|\phi_{\mathrm{I}}\right\rangle=\sum_{s^{\prime}=0}^{s} \frac{\alpha_{z}^{s-s^{\prime}}}{\sqrt{\left(s-s^{\prime}\right) !}}\left|\phi_{\mathrm{I}}^{s^{\prime}}\right\rangle, \quad\left|\phi_{\mathrm{II}}\right\rangle=\sum_{s^{\prime}=0}^{s-2} \frac{\alpha_{z}^{s-2-s^{\prime}}}{\sqrt{\left(s-2-s^{\prime}\right) !}}\left|\phi_{\mathrm{II}}^{s^{\prime}}\right\rangle, \\
\left|\phi_{\mathrm{I}, \mathrm{II}}^{s^{\prime}}\right\rangle=\frac{1}{s^{\prime} !} \alpha^{a_{1}} \ldots \alpha^{a_{s^{\prime}}} \phi_{\mathrm{I}, \mathrm{II}}^{a_{1} \ldots a_{s^{\prime}}}|0\rangle .
\end{gathered}
$$

В терминах скалярных, векторных и бесследовых тензорных полей лагранжиан (4.30) может быть представлен следующим образом:

$$
\begin{aligned}
\mathcal{L}_{\text {tot }} & =\sum_{s^{\prime}=0}^{s} \mathcal{L}_{\mathrm{I}}^{s^{\prime}}-\sum_{s^{\prime}=0}^{s-2} \mathcal{L}_{\mathrm{II}}^{s^{\prime}}+\sum_{s^{\prime}=0}^{s-1} \mathcal{L}_{\mathrm{FP}}^{s^{\prime}}, \\
\mathcal{L}_{\mathrm{I}}^{s^{\prime}} & =\frac{1}{2 s^{\prime} !} \phi_{\mathrm{I}}^{a_{1} \ldots a_{s^{\prime}}} \square^{\nu_{s^{\prime}}} \phi_{\mathrm{I}}^{a_{1} \ldots a_{s^{\prime}}}, \quad \mathcal{L}_{\mathrm{II}}^{s^{\prime}}=\frac{1}{2 s^{\prime} !} \phi_{\mathrm{II}}^{a_{1} \ldots a_{s^{\prime}}} \square^{\nu_{s^{\prime}}+2} \phi_{\mathrm{II}}^{a_{1} \ldots a_{s^{\prime}}}, \\
\mathcal{L}_{\mathrm{FP}}^{s^{\prime}} & =\frac{1}{s^{\prime} !} \bar{c}^{a_{1} \ldots a_{s^{\prime}}} \square^{\nu_{s^{\prime}}+1} c^{a_{1} \ldots a_{s^{\prime}}} .
\end{aligned}
$$

Из выражений (4.32) видно, что вакуумная статистическая сумма дается выражениями

$$
\begin{aligned}
Z & =\prod_{s^{\prime}=0}^{s-1}\left(D^{s^{\prime}} D^{s^{\prime}}\right)^{\nu_{s^{\prime}}+1}\left(\prod_{s^{\prime}=0}^{s}\left(D^{s^{\prime}}\right)^{\nu_{s^{\prime}}} \prod_{s^{\prime}=0}^{s-2}\left(D^{s^{\prime}}\right)^{\nu_{s^{\prime}}+2}\right)^{-1}, \\
D^{s^{\prime}} & \equiv(\operatorname{det}(-\square))^{1 / 2},
\end{aligned}
$$

где детерминант оператора Лапласа вычисляется на пространстве бесследового тензорного поля ранга $s^{\prime}$. Приводя множители в числителе и знаменателе в (4.33), получим следующее выражение для вакуумной статистической суммы:

$$
Z=\frac{\left(D^{s-1}\right)^{\nu_{s}+1}}{\left(D^{s}\right)^{\nu_{s}}}, \quad \nu_{s}=s+\frac{d-4}{2}
$$

Статсумма (4.35) была получена ранее другими методами в работах [37], [14]. В работе [14] было также замечено, что при использовании соотношения

$$
D^{s^{\prime}}=D^{s^{\prime} \perp} D^{s^{\prime}-1},
$$


где $D^{s^{\prime} \perp}$ имеет тот же вид (4.34), однако теперь детерминант оператора Лапласа вычисляется на пространстве бесследового и бездивергентного тензорного поля ранга $s^{\prime}$, статистическая сумма (4.35) может быть преобразована к виду

$$
Z=\frac{1}{\left(D^{s \perp}\right)^{(d-4) / 2}} \prod_{s^{\prime}=0}^{s-1} \frac{D^{s^{\prime} \perp}}{D^{s \perp}} .
$$

В работе [14] выражение (4.37) было обобщено на случай статистической суммы конформного поля в пространстве АдС. Вычисление статистической суммы конформного поля в пространстве АдС различными методами можно найти в работах [14], [38].

\section{Список принятых обозначений}

ПРИЛОЖЕНИЕ

Векторные индексы алгебры $s o(d, 1)$ принимают значения $A, B, C, E=0,1, \ldots, d$, а векторные индексы алгебры $s o(d-1,1)$ принимают значения $a, b, c, e=0,1, \ldots, d-1$. Чтобы упростить выражения, мы опускаем плоские метрики $\eta^{A B}=(-,+, \ldots,+)$ и $\eta^{a b}=(-,+, \ldots,+)$ в скалярных произведениях: $X^{A} Y^{A} \equiv \eta_{A B} X^{A} Y^{B}, X^{a} Y^{a} \equiv$ $\eta_{a b} X^{a} Y^{b}$.

Ковариантная производная $D^{A}$ определяется соотношениями $D^{A}=\eta^{A B} D_{B}$,

$$
D_{A} \equiv e_{\frac{m}{A}} D_{\underline{m}}, \quad D_{\underline{m}} \equiv \partial_{\underline{m}}+\frac{1}{2} \omega_{\underline{m}}^{A B} M^{A B}, \quad M^{A B}=\alpha^{A} \bar{\alpha}^{B}-\alpha^{B} \bar{\alpha}^{A},
$$

$\partial_{\underline{m}}=\partial / \partial x^{\underline{m}}, \underline{m}=0,1, \ldots, d$, где $x^{\underline{m}}-$ координаты в пространстве $(\mathrm{A})$ дС ${ }_{d+1}, e^{\frac{m}{A}}-$ обратная матрица к базису реперов $e_{\underline{m}} A, e^{\frac{m}{A}} e_{\underline{m} B}=\eta_{A B}, D_{\underline{m}}$ - ковариантная производная со связностью Лоренца $\omega_{m}^{A B}$, а $M^{A B}$ - оператор спина алгебры Лоренца $s o(d, 1)$. Тензорное поле в пространстве $(\mathrm{A}) д С$ с плоскими индексами $\Phi^{A_{1} \ldots A_{s}}$ связано с контравариантным тензорным полем $\Phi \underline{m}_{1} \ldots \underline{m}_{s}$ стандартным образом: $\Phi^{A_{1} \ldots A_{s}} \equiv$ $e_{\underline{m}_{1}}^{A_{1}} \ldots e_{\underline{m}_{s}}^{A_{s}} \Phi \underline{m}_{1} \ldots \underline{m}_{s}$. Оператор Даламбера в пространстве (А)дС определяется соотношением

$$
\square_{(\mathrm{A}) \mathrm{d} S} \equiv D^{A} D^{A}+\omega^{A A B} D^{B}, \quad \omega^{A B C} \equiv e^{A \underline{m}} \omega_{\underline{m}}^{B C}, \quad e \equiv \operatorname{det} e_{\underline{m}}^{A} .
$$

Производная $D_{\underline{m}}(П .1)$ действует на кет-векторы, построенные из осцилляторов $\alpha^{A}$. Производные, действующие непосредственно на поля, обозначаются как $\mathcal{D}_{\underline{m}}$. Действие такой производной на векторное поле с плоскими индексами определяется соотношением

$$
\mathcal{D}_{\underline{m}} \phi^{A}=\partial_{\underline{m}} \phi^{A}+\omega_{\underline{m}}^{A B}(e) \phi^{B} .
$$

Вместо $\mathcal{D}_{\underline{m}}$ мы предпочитаем использовать производную с плоскими индексами $\mathcal{D}^{A}$,

$$
\mathcal{D}_{A} \equiv e^{\frac{m}{A}} \mathcal{D}_{\underline{m}}, \quad \mathcal{D}^{A}=\eta^{A B} \mathcal{D}_{B}, \quad\left[\mathcal{D}^{A}, \mathcal{D}^{B}\right] \phi^{C}=R^{A B C E} \phi^{E},
$$

$\mathcal{D}^{2} \equiv \mathcal{D}^{A} \mathcal{D}^{A}$, где тензор Римана пространства (А)дС имеет вид

$$
\begin{aligned}
R^{A B C E} & =\rho\left(\eta^{A C} \eta^{B E}-\eta^{A E} \eta^{B C}\right), \quad \rho=\frac{\epsilon}{R^{2}}, \\
\epsilon & = \begin{cases}1 & \text { для пространства дС, } \\
-1 & \text { для пространства АдС. }\end{cases}
\end{aligned}
$$


Операторы рождения $\alpha^{A}, \zeta$ и соответствующие операторы уничтожения $\bar{\alpha}^{A}, \bar{\zeta}$ называются осцилляторами. Коммутационные соотношения, вакуум $|0\rangle$ и правила эрмитова сопряжения определяются соотношениями

$$
\left[\bar{\alpha}^{A}, \alpha^{B}\right]=\eta^{A B}, \quad[\bar{\zeta}, \zeta]=1, \quad \bar{\alpha}^{A}|0\rangle=0, \quad \bar{\zeta}|0\rangle=0, \quad \alpha^{A \dagger}=\bar{\alpha}^{A}, \quad \zeta^{\dagger}=\bar{\zeta}
$$

Осцилляторы $\alpha^{A}, \bar{\alpha}^{A}$ и $\zeta, \bar{\zeta}$ преобразуются соответственно в векторных и скалярных представлениях алгебры $s o(d, 1)$. Производные от координат $x^{a}, z$ обозначаются как $\partial^{a} \equiv \eta^{a b} \partial / \partial x^{b}, \partial_{z} \equiv \partial / \partial z$. В базисе $s o(d-1,1)$-алгебры используем разложения осцилляторов $\alpha^{A}=\alpha^{a}, \alpha^{z}$ и плоской метрики $\eta^{A B}=\eta^{a b}, \eta^{z z}$, где $\eta^{z z}=1$. Используются следующие сокращения для скалярных произведений осцилляторов и производных:

$$
\begin{array}{cccc}
\boldsymbol{\alpha} \mathbf{D} \equiv \alpha^{A} D^{A}, & \overline{\boldsymbol{\alpha}} \mathbf{D} \equiv \bar{\alpha}^{A} D^{A}, & \boldsymbol{\alpha}^{2} \equiv \alpha^{A} \alpha^{A}, & \overline{\boldsymbol{\alpha}}^{2} \equiv \bar{\alpha}^{A} \bar{\alpha}^{A}, \\
\alpha \partial \equiv \alpha^{a} \partial^{a}, & \bar{\alpha} \partial \equiv \bar{\alpha}^{a} \partial^{a}, & \alpha^{2} \equiv \alpha^{a} \alpha^{a}, & \bar{\alpha}^{2} \equiv \bar{\alpha}^{a} \bar{\alpha}^{a}, \\
N_{\boldsymbol{\alpha}} \equiv \alpha^{A} \bar{\alpha}^{A}, & N_{\alpha} \equiv \alpha^{a} \bar{\alpha}^{a}, & N_{\zeta} \equiv \zeta \bar{\zeta}, & N_{z} \equiv \alpha^{z} \bar{\alpha}^{z}, \\
\Pi^{[1,2]} \equiv 1-\alpha^{2} \frac{1}{2\left(2 N_{\alpha}+d\right)} \bar{\alpha}^{2}, & \boldsymbol{\Pi}^{[1,2]} \equiv 1-\boldsymbol{\alpha}^{2} \frac{1}{2\left(2 N_{\boldsymbol{\alpha}}+d+1\right)} \overline{\boldsymbol{\alpha}}^{2}, \\
\square \equiv \partial^{a} \partial^{a}, \quad \mu \equiv 1-\frac{1}{4} \alpha^{2} \bar{\alpha}^{2}, \quad \boldsymbol{\mu} \equiv 1-\frac{1}{4} \boldsymbol{\alpha}^{2} \overline{\boldsymbol{\alpha}}^{2} .
\end{array}
$$

Бра- и кет-векторы полей Фаддеева-Попова связаны соотношениями $\langle c|=| c\rangle^{\dagger}$, $\langle\bar{c}|=-| \bar{c}\rangle^{\dagger}$, а для тензорных полей Фаддеева-Попова используем следующие правила эрмитова сопряжения: $c^{A_{1} \ldots A_{s^{\prime}} \dagger}=c^{A_{1} \ldots A_{s^{\prime}}}, \bar{c}^{A_{1} \ldots A_{s^{\prime}} \dagger}=-\bar{c}^{A_{1} \ldots A_{s^{\prime}}}$.

Благодарности. Работа поддержана РФФИ (грант № 14-02-01171).

\section{Список литературы}

[1] А. А. Славнов, ТМФ, 10:2 (1972), 153-161; J. C. Taylor, Nucl. Phys. B, 33:2 (1971), 436-444.

[2] C. Becchi, A. Rouet, R. Stora, Ann. Phys., 98:2 (1976), 287-321; И. В. Тютин, Калибровочная инвариантность в теории поля и статистической физике в операторном формализме, Препринт № 35, ФИАН, М., 1975, arXiv: 0812.0580.

[3] D. Z. Freedman, S. D. Mathur, A. Matusis, L. Rastelli, Nucl. Phys. B, 546:1-2 (1999), 96-118, arXiv: hep-th/9804058.

[4] H. Liu, A. A. Tseytlin, Nucl. Phys. B, 533:1-3 (1998), 88-108, arXiv: hep-th/9804083.

[5] W. Mück, K.S. Viswanathan, Phys. Rev. D, 58:10 (1998), 106006, 7 pp., arXiv: hep-th/9805145; A. Polishchuk, JHEP, 07 (1999), 007, 13 pp., arXiv: hep-th/9905048.

[6] R. R. Metsaev, Phys. Rev. D, 83:10 (2011), 106004, 28 pp., arXiv: 1011.4261.

[7] R. R. Metsaev, Phys. Rev. D, 81:10 (2010), 106002, 29 pp., arXiv: 0907.4678.

[8] R. R. Metsaev, Phys. Rev. D, 85:12 (2012), 126011, 20 pp., arXiv: 1110.3749.

[9] V. K. Dobrev, Nucl. Phys. B, 553:3 (1999), 559-582, arXiv: hep-th/9812194; N. Aizawa, V.K. Dobrev, Intertwining operator realization of anti de Sitter holography, arXiv: 1406.2129.

[10] R. R. Metsaev, Nucl. Phys. B, 563:1-2 (1999), 295-348, arXiv: hep-th/9906217.

[11] R. R. Metsaev, Phys. Lett. B, 636:3-4 (2006), 227-233, arXiv: hep-th/0512330.

[12] R. R. Metsaev, Phys. Rev. D, 78:10 (2008), 106010, 31 pp., arXiv: 0805.3472.

[13] R. K. Gupta, S. Lal, JHEP, 07 (2012), 071, 16 pp., arXiv: 1205.1130.

4 Теоретическая и математическая физика, т. 181, № 3, 2014 г. 
[14] A. A. Tseytlin, Nucl. Phys. B, 877:2 (2013), 598-631, arXiv: 1309.0785.

[15] R. R. Metsaev, Phys. Lett. B, 671:1 (2009), 128-134, arXiv: 0808.3945.

[16] R. R. Metsaev, Phys. Lett. B, 682:4-5 (2010), 455-461, arXiv: 0907.2207.

[17] C. Fronsdal, Phys. Rev. D, 20:4 (1979), 848-856.

[18] I. L. Buchbinder, A. Pashnev, M. Tsulaia, Phys. Lett. B, 523:3-4 (2001), 338-346, arXiv: hep-th/0109067.

[19] Yu. M. Zinoviev, "On massive high spin particles in (A)d", arXiv: hep-th/0108192.

[20] V. E. Lopatin, M. A. Vasiliev, Modern Phys. Lett. A, 3:3 (1988), 257-270; Yu. M. Zinoviev, Nucl. Phys. B, 808:1 (2009), 185-204, arXiv: 0808.1778; D. S. Ponomarev, M. A. Vasiliev, Nucl. Phys. B, 839:3 (2010), 466-498, arXiv: 1001.0062.

[21] I. L. Buchbinder, V.A. Krykhtin, P. M. Lavrov, Nucl. Phys. B, 762:3 (2007), 344-376, arXiv: hep-th/0608005.

[22] K. B. Alkalaev, M. Grigoriev, Nucl. Phys. B, 835:1-2 (2010), 197-220, arXiv: 0910.2690.

[23] K. Alkalaev, M. Grigoriev, Nucl. Phys. B, 853:3 (2011), 663-687, arXiv: 1105.6111.

[24] M. Grigoriev, A. Waldron, Nucl. Phys. B, 853:2 (2011), 291-326, arXiv: 1104.4994.

[25] N. Boulanger, S. Leclercq, P. Sundell, JHEP, 08 (2008), 056, 33 pp., arXiv: 0805.2764.

[26] R. R. Metsaev, Phys. Lett. B, 720:1-3 (2013), 237-243, arXiv: 1205.3131.

[27] M. Henneaux, G. L. Gomez, R. Rahman, JHEP, 08 (2012), 093, 44 pp., arXiv: 1206.1048; 01 (2014), 087, 44 pp., arXiv: 1310.5152.

[28] M. Taronna, JHEP, 04 (2012), 029, 75 pp., arXiv: 1107.5843.

[29] P. Dempster, M. Tsulaia, Nucl. Phys. B, 865:2 (2012), 353-375, arXiv: 1203.5597.

[30] I. L. Buchbinder, P. Dempster, M. Tsulaia, Nucl. Phys. B, 877:2 (2013), 260-289, arXiv: 1308.5539 .

[31] I. A. Batalin, K. Bering, P. M. Lavrov, I. V. Tyutin, A systematic study of finite field dependent BRST-BV transformations in $S p(2)$ extended field-antifield formalism, arXiv: 1406.4695; P. Y. Moshin, A. A. Reshetnyak, Field-dependent BRST-antiBRST Lagrangian transformations, arXiv: 1406.5086.

[32] G. E. Arutyunov, S. A. Frolov, Nucl. Phys. B, 544:3 (1999), 576-589, arXiv: hep-th/9806216.

[33] V.E. Didenko, E. D. Skvortsov, JHEP, 04 (2013), 158, 27 pp., arXiv: 1210.7963; O. A. Gelfond, M. A. Vasiliev, Nucl. Phys. B, 876:3 (2013), 871-917, arXiv: 1301.3123; V.E. Didenko, J. Mei, E. D. Skvortsov, Phys. Rev. D, 88:4 (2013), 046011, 10 pp., arXiv: 1301.4166.

[34] I. Y. Aref'eva, I. V. Volovich, On large $N$ conformal theories, field theories in anti-De Sitter space and singletons, arXiv: hep-th/9803028.

[35] X. Bekaert, M. Grigoriev, Nucl. Phys. B, 876:2 (2013), 667-714, arXiv: 1305.0162; J. Phys. A: Math. Theor., 46:21 (2013), 214008, 23 pp., arXiv: 1207.3439.

[36] M. A. Vasiliev, J. Phys. A, 46:21 (2013), 214013, 47 pp., arXiv: 1203.5554.

[37] E. S. Fradkin, A. A. Tseytlin, Phys. Rep., 119:4-5 (1985), 233-362.

[38] R. R. Metsaev, Nucl. Phys. B, 885 (2014), 734-771, arXiv: 1404.3712. 